\title{
Optimal Enterprise Risk Management and Decision Making with Shared and Dependent Risks
}

\section{Introduction}

Dynamic enterprise risk management (ERM) entails holistic decision-making for an integrated firm with multiple business divisions. In particular, risk management plays an essential role in the decision process as it is naturally connected to other critical corporate functions (e.g., capital budgeting, asset-liability matching) via shared and dependent risk exposures and synergetic relationships within the corporate structure. As companies grow larger and more diverse, interactive complexity increases so no individual organizational component (such as a business division) can be easily insulated. Complex and tightly coupled corporate systems can be highly susceptible to catastrophic breakdowns as evident by the financial services conglomerate AIG during the financial crisis wherein realized risks in the Financial Products division spilled to other divisions and flowed upward to the holding company. One of the ultimate goals of the emerging ERM practice (cf., COSO 2004) is to facilitate companies in overall decision-making in light of these complexities to maximize firm value. Motivated by this challenge, this study describes the ERM decision process in an optimization framework using a copula-based decision tree model to accommodate a flexible dynamic dependence structure among risks, a key to ERM success.

Largely driven by regulatory forces (e.g., The Sarbanes-Oxley Act of 2002) and rating agencies (e.g., S\&P ERM ratings since 2006), ERM has grown considerably in the last decade (Beasley et al. 2012, Deloitte 2012). The 2013 RIMS Enterprise Risk Management Survey of 1000+ risk professionals found 21 percent of surveyed entities had fully integrated ERM across the organization and 42 percent had partially integrated ERM, for an overall ERM adoption rate of $63 \%$, double that of 2009 (RIMS 2013). ERM practice is especially prominent among financial institutions and the trend is continuing. For example, NAIC's new regulatory standard "Own Risk and Solvency Assessment (ORSA)" is expected to provide yet another major push toward advancing the commitment to ERM as a value-enhancing strategy for U.S. insurers at all levels.

Benefits of integrated risk management have been emphasized in the literature (e.g., Beasley et al. 2008, Hoyt and Liebenberg 2011, Pagach and Warr 2011, Lin et al. 2012, Grace et al. 2015). We refer the interested readers to Gatzert and Martin (2015) for a comprehensive literature review. While risk management decisions should be contemplated at the enterprise level and integrated with other corporate functions considering dependencies (rather than modeled separately),only limited research has addressed the challenge of optimally coordinating corporate decision making through ERM (e.g., MacMinn 1987, Ai et al. 2012, Lin et al. 2012, Gatzert and Kolb 2014). We approach this problem by jointly considering different corporate functions under a single ERM decision framework, following the spirit of seminal works by Froot et al. (1993), Froot and Stein (1998), and Froot (2007). 
Dependence modeling is a core component when formulating a corporate ERM decision making process. Traditional corporate risk management often addresses risks in "silos," overlooking possible dependence and hence the collective contribution to overall corporate performance. As noted by Obersteadt (2013, pp.54), "The decision to implement ERM is partly driven by the recognition that risks are interrelated and interdependent. Relying on the traditional approach of managing individual risks separately in their own risk silos tends too often lead to serious and systematic errors in risk identification and assessment." As we show in our analysis, neglecting dependencies can result in significant deviations from optimal enterprise wide strategic plans, potentially leading to catastrophic outcomes (in face of high positive dependence) and/or over-hedging in situations when "natural hedging" opportunities are ignored. However, dependencies are often neglected due to computational challenges as discussed in Smith et al. (1992), Gollier (2001), Embrechts et al. (2002), Tsetlin and Winkler (2005), Ai et al. (2012), and Wang et al. (2015). Here we explicitly incorporate modeling of dependence among risks in our optimized ERM decision making framework with a copula-based measure. In particular, through the choice of the copula function (e.g., normal, t, or Archimedean copulas) and the correlation measure (e.g., Pearson product moment correlation, Spearman's rank-order correlation or Kendall's tau correlation), we can accommodate both linear and nonlinear dependence structure amenable to the practical applications at hand.

This paper focuses on the integration of risk management and capital budgeting decision-making processes 1 recognizing both shared risks across enterprise components and dependent risks within the firm 2 We provide a framework where two sets of decisions are made in a single enterpriselevel optimization problem, taking account of capital requirements, cash flow potentials, and risk exposures within each business division and their inter-dependencies. The optimization problem is constructed via the intuitive and visual interface of a decision tree to provide a clear depiction of the contingent planning process, and dependencies are directly modeled using a copula-based measure. We illustrate the framework using a hypothetical financial services company and subsequently discuss model insights for practice.

The paper is organized as follows. Section 2 describes our integrated ERM (and capital budgeting) decision framework, and the strengths and weaknesses of the modeling approach. Section 3 gives a detailed implemented illustration for a hypothetical financial services company. Extensions and further discussions are provided in Section 4, and Section 5 concludes the paper.

\footnotetext{
${ }^{1}$ In the insurance literature in particular, much more attention is devoted to capital allocation models than capital budgeting. An optimization model is often constructed for capital allocation based on appropriate risk measures with dependence considerations (e.g., Laeven and Goovaerts 2004). Myers and Read (2001) shows how option pricing methods can be used in capital allocation for insurance companies. It is worth noting, however, that capital budgeting decision-making can be an entirely separate optimization process that does not trivially relate to the capital allocation process. See Gründl and Schmeiser (2007) for a review and comparison of different capital allocation models, and a discussion of problems arising from using capital allocation models for making budgeting decisions.

${ }^{2}$ Brealey and Myers (2003) and Bernardo et al. (2004) note that the majority of corporate investments are financed by internal funds. Therefore, our current formulation focuses on capital budgeting using internal capital markets and not explicitly considering external financing policies.
} 


\section{The Integrated Corporate ERM Decision Framework}

The integrated enterprise risk management and capital budgeting framework presented here considers a multi-divisional corporation with a multi-period capital budgeting horizon. The framework is formulated as an optimization problem for the decision maker, where resources are allocated to projects in different divisions in light of shared and dependent risks within and across these divisions while simultaneously determining corporate risk management strategies. We assume that utility maximization of the corporate decision maker (obtained by solving the optimization problem) also achieves the corporation's strategic goal of shareholder value maximization.3 Our use of the copula dependence modeling approach allows for a large class of marginal risk and return relationships as well as allowing varying linear and nonlinear dependence structures.

A decision tree model provides an intuitive and visual interface useful for constructing an optimization ERM decision framework. Decision trees are commonly used in risk and decision analysis to identify optimal dynamic strategies in the presence of uncertainties (Clemen and Reilly 1999, Sherali et al. 2011, and Wang and Dyer 2012). The strengths and robustness of the decision tree approach are documented and discussed in the literature (e.g., Clemen and Reilly 1999). While decision tree analysis has become a fundamental tool for decision-making under uncertainties in industrial development contexts, it is less often used in corporate risk management and financial decision-making. This may be due to the exponentially growing tree size (and corresponding computational difficulty) in complicated settings with multiple sources of dependent uncertainties and sequential decisions. Here we adapt a dimension reduction technique (Gustafsson and Salo 2005), coupled with a discretized version of copula analysis, to address this computational issue.

A decision tree builds upon fully described risk events and delineates all possible paths a decision maker might follow over time, including all decision alternatives and the corresponding outcomes under each risk event. In formulating our model so as to address several challenges associated with practical ERM implementation, we extend the statistical technique in Wang and Dyer (2012) to build a copula-based dependent decision tree. In our model, risk dependences throughout the capital budgeting horizon are captured with a dependent "state of nature" tree, reflecting both the individual risk characteristics and inter-relations of these risks within and across business divisions and time periods. Cash flows of the corporate portfolio are characterized within the dependent tree structure to reflect risks, investment opportunities, decisions, and capital constraints. The corporate decision maker's risk preferences summarized by a utility function are then optimized to simultaneously obtain capital budgeting decisions and risk management strategies.

\subsection{Utility Maximization in the Objective Function}

In our model the corporate decision maker maximizes the certainty equivalent $(\mathrm{CE})$ measure of her expected utility derived from the corporation's capital resource positions in each of the states of nature at the end of the capital budgeting horizon. We assume a classic mean-risk model for the

\footnotetext{
${ }^{3}$ It has been long noted (Pencavel 1979 and Deaton 1981), that after tax profit maximization (shareholder wealth) is consistent with this utility maximization approach when there is a progressive tax structure.
} 
von Neumann-Morgenstern utility function.

The expected future capital resource position at the end of the capital budgeting horizon $t=$ $T$ is:

$$
E V_{T}=\sum_{s \in S_{T}} p(s) V_{s}
$$

where $V_{s}$ is the capital resource position in state $s$ resulting from one or more corporate risk exposures, $p(s)$ is the associated probability, and $S_{T}$ is the set of all possible states at $T$. In our applications the corporate states of nature will be represented utilizing a tree-form development detailed in Section 2.2.

While the literature provides a broad range of available risk measures that can be used in this general framework (cf., Natarajan et al. 2009), here we focus on the lower semi-absolute deviation measure (LSAD) (e.g., Fishburn 1977 and Eppen et al. 1989) to emphasize the downside risk that is usually more of a concern (Gustafsson and Salo 2005 and Ang et al. 2006). Additionally, the LSAD risk measure has desirable theoretic properties such as its consistency with the first and second order stochastic dominance (Fishburn 1977). The LSAD risk measure is given by

$$
L S A D_{T}=\sum_{s \in S_{T D}} p(s)\left|V_{s}-E V_{T}\right|
$$

where the summation is extended only over the states in $S_{T D}=\left\{s: V_{s}<E V_{T}\right\}$. Under the assumption that the decision maker's utility function is a classic von Neumann-Morgenstern utility function, the objective function can be written as

$$
\text { Maximize } C E=\left[E V_{T}-\lambda \times L S A D_{T}\right]
$$

where $E V_{T}$ is defined by Equation (1), $L S A D_{T}$ is defined by Equation (2), and $\lambda$ is the risk aversion coefficient (Gustafsson and Salo 2005). Note that the mean-risk framework of our approach is general in nature, and other utility functions and risk measures can be reasonably chosen to formulate our model in a very similar way ${ }^{4}$

\subsection{Utilizing Tree Construction and Modeling Dependencies in Dynamic States of Nature}

Expected future project cash flows are contingent on corporate states of nature, which in turn are driven by multiple sources of dependent risks across different business divisions and time. It is crucial to properly model dependence among these risk exposures as they are central to the idea of ERM. We describe depending modeling in our framework in this section.

\footnotetext{
${ }^{4}$ In particular, we could use other risk measures such as variance. Instead of calculating the LSAD, we would alternatively calculate the variance of the final capital resource positions and construct a Sharpe-Ratio type of objective function. This will result in a non-linear optimization problem for which solution techniques are available. We refer the interested readers to Gustafsson and Salo (2005) for more detailed discussion on general mean-risk models in the Contingent Portfolio Programming framework.
} 


\subsubsection{Overview of the decision tree approach and its strengths}

From a modeling perspective, our problem formulation is essentially a stochastic optimization with managerial flexibilities under dependent future uncertainties. We have chosen to formulate the problem using a decision tree approach. Other competing model formulations include continuous time option pricing methods and Monte Carlo stochastic simulation methods. While we have not found any comparisons of these approaches in the (enterprise) risk management literature, there have been such comparisons in the finance and management science literature. In the finance literature managerial flexibilities are considered as providing real options to the investor. Real option valuation approaches extend option pricing theory to situations where flexibility in the deployment of real assets increases the value of a project. For example, in our proposed framework, the option of starting a project is analogous to a call option and the option of discontinuing a project is analogous to a put option with a strike price equal to the capital investment required for the project. Other examples of managerial flexibilities include the options to switch, to defer, to expand, or take other actions on alternative capital investment.

In the earlier literature, existing financial options pricing methods were directly applied to solve these real options problems. For instance, Myers and Read (2001) shows how option pricing methods can be used in capital allocation of insurance companies. However, these standard option pricing-based capital allocation methods are difficult to apply in problems with multiple underlying uncertainties and American style early exercise real options. Instead, decision trees and Monte Carlo simulation have been proposed for real options valuation. Monte Carlo simulation is computationally tractable for European style options, but it is more difficult to implement for complex options with large numbers of alternatives and early exercise dates.5 Monte Carlo methods and more generally numerical integration methods essentially approximate the values of complicated functions by a weighted sum of discrete values from a large number of simulated scenarios. Large simulation models can suffer from "the curse of dimensionality" as the multivariate dependence structure is simulated and carried forward in the computation. The decision tree model approach provides one algorithm for representing multivariate dependencies, wherein continuous uncertainties are discretized to simplify the tree-rollback procedure and the computational requirements. In many cases, it is more economical to carefully choose a small set of input values, rather than generate a large number of random scenarios that "cover the waterfront" via Monte Carlo simulation. We believe that the decision tree-based ERM decision framework is a case that provides a solution to the tradeoff between parsimonious representation of the dependence structure and computational feasibility of the model.

In addition to easing the computational burden, discretization in decision analysis also aids in understanding of the problem by defining distinct scenarios, and can simplify the work of assessing

\footnotetext{
${ }^{5}$ Longstaff and Schwartz (2001) provided a hybrid approach of simulation and regression to overcome the early exercise issue. However, the computational complexity is still challenging with increased number of decisions and alternatives (Smith 2005).
} 
a probability distribution supplied by a decision-maker or subject matter expert (cf. Clemen et al. 2000). An additional important advantage of our tree-based discretization method is it is easily explained, whereas simulation methods are often viewed as black boxes by decision makers. Finally, the tree method can incorporate constraints into an optimization framework.

Decision tree models have been shown to perform well compared to option pricing models and Monte Carlo simulations. Smith and Nau (1995) show that the decision tree approach is an equivalent alternative to the option pricing method and can further extend option pricing techniques to real option problems with incomplete securities markets. Clemen and Reilly (1999) compared the results of the copulas-based multivariate discrete decision tree approach with Monte Carlo simulation approach. They showed that "the discrete approximation provides a reasonable estimate of the expected value (with a difference of less than 1.53\%), the standard deviation (a difference of less than $0.38 \%$ ), and a very reasonable fit to the tails of the copula-based risk profile" (pp.218). In this paper, we adopt the Wang and Dyer (2012) copula-based multivariate dependent decision tree approach that is mathematically equivalent to yet computationally more efficient than the Clemen and Reilly (1999) approach. The comparisons shown in Clemen and Reilly (1999) and more recently in Wang et al. (2015) help verify the satisfactory performance of decision tree models. For a further discussion of the relative benefits of using a decision tree approach versus a simulation approach for real options and other related problems, see Clemen and Reilly (1999) and Wang and Dyer (2010, 2012).

\subsubsection{Discretization in the decision tree approach and its robustness}

Decision tree is a popular modeling tool, but can represent only discrete scenarios. Therefore, it is necessary to approximate continuous uncertainties with properly designed discrete uncertainties, a process known as discretization. The extended Pearson-Tukey (henceforth EP-T; Keefer and Bodily 1983) method and other discretization methods have been proposed to approximate important properties of the corresponding continuous distribution, often with carefully chosen percentile values and associated probabilities, and typically attempts to match the first, second and other central moments of the continuous distribution (cf. Hammond and Bickel 2013). In this paper, we use the widely recognized EP-T method for discretizing continuous distributions, where each risk realization is represented with a trinomial approximation of all possible scenarios. Throughout the decision tree structure a constant set of pre-optimized probabilities $(0.185,0.630$, and 0.185$)$ is assigned to the point risk realizations in each of the three scenarios ("up", "middle" and "down") corresponding to the $5^{t h}, 50^{t h}$, and $95^{t h}$ percentiles of the continuous conditional distributions.

The EP-T method has been shown to be a robust discretization approach in the literature. Keefer and Bodily (1983) systematically examine the performance of several popular discretization methods including lognormal distributions and a set of right-skewed, left-skewed, $\bigcap$-shaped, and J-shaped beta distributions, generally representing a large set of distribution families. They found the EP-T method performed the best with respect to preserving the moments of the underlying distribution. Keefer (1994) show that EP-T method is accurate in representing expected utilities or certainty equivalents over a large variety of test distributions when the level of risk aversion 
and the characteristics of the distributions are within reasonable bounds. In addition, Keefer and Bodily (1983) also examine the EP-T method in multivariate contexts and confirms the accuracy of the EP-T method in approximating the mean, variance and covariance of the multivariate joint distributions over a large set of the marginal distributions. More recently, Hammond (2014) extends previous research by considering a wider range of distributions, through the use of the Pearson and Johnson distribution systems, which cover several commonly-used distribution families. He also confirms that the EP-T approach closely approximate the moments of a wide range of distributions.

In this paper we apply the EP-T method because of its robustness shown in the literature, however it should be noted that it would be straight forward to apply other discretization methods within our modeling approach. Additionally, while the three-point approximations (as used in the EP-T method) is common practice (to achieve a balance between sufficient approximation capability and managerial and computational tractability), the proposed framework is also applicable to more general approximations methods such as the five-point approximation methods discussed in Smith (1993) and Hammond (2014). For a recent and detailed review of the literature on the alternative discretization schemes, see Hammond and Bickel (2013) and Hammond (2014).

\subsubsection{Copulas-based dependent decision tree approach}

Modeling managerial flexibility under multivariate dependent uncertainties often arises as a key issue in enterprise-wide decision-making for capital budgeting and risk management. Following the recent finance and risk management literature (e.g., Cherubini et al. 2004, Zhang and Dukic 2012) dependencies are directly modeled using a copula-based measure. A copula allows a joint distribution of random variables $F\left(X_{1}, \ldots, X_{n}\right)=C\left(F_{1}\left(X_{1}\right), \ldots, F_{n}\left(X_{n}\right)\right)$ to be expressed as a function of the marginal distributions $F\left(X_{1}\right), \ldots, F\left(X_{n}\right)$, and fully captures the dependence structure among the uncertainties through the choice of the copula function $C($.$) and the dependence measure. Our$ proposed framework allows the use of multiple dependence measures within parametric families of copulas such as normal copulas, t-copulas and Archimedean copulas, with inputs including the usual Pearson product moment linear correlation, rank order correlations and tail dependence. For instance, a normal copula function underlies the multivariate normal distribution and shares the multivariate normal distribution dependence structure while allowing arbitrary marginal distributions for the uncertainties with either product moment correlations or rank correlations as inputs to measure dependence (Embrechts et al. 2003) 6 The $t$-copulas and Archimedean copulas can also capture extreme co-movements in the lower and upper tails of the distributions, e.g., tail dependence. See Frees and Valdez (1998), Nelson (1999) and Embrechts (2009) for an excellent introduction and review of copulas.

Under our modeling approach an optimization problem is constructed using a decision tree to provide a clear illustration of the contingent planning process. Copula modeling is used to incorporate dependencies and we extend the dependent decision tree approach proposed by Wang and Dyer (2012) to formulate an optimization problem into a standard decision tree approach. Using only

\footnotetext{
${ }^{6}$ An important property of the Spearman's and Kendall's rank correlation measures of dependence is that they capture nonlinear dependencies that product moment correlations cannot.
} 
information of marginal distributions and correlation inputs, this approach allows multiple dependent risks with arbitrary marginal distributions to be represented in a decision tree with a sequence of discretized conditional probability distributions. This approach provides better modeling flexibility than alternative approaches (Clemen and Reilly 1999) and supports popular copula families, such as elliptical copulas including the widely used normal copula and $t$-copula and Archimedean copulas. Additionally, it is mathematically more tractable and computationally more efficient. We briefly illustrate this approach in Figure 1.

On the left side of Figure 1, the state of nature tree outlines possible contingent realizations of two dependent uncertainties. It starts with a single base state $S_{0}$ at time 0 , leading to a set of three states at time $1, S_{1}, S_{2}, S_{3}$, corresponding to the three possible outcomes ("up", "middle" and "down") per the EP-T trinomial approximation of the first uncertainty. Likewise, for the second uncertainty, a set of three subsequent states representing three contingent outcomes follows each realization of the preceding state of uncertainty. The unconditional probabilistic outcome in each state is computed recursively from the conditional outcomes of the preceding states and thus the final set of nine outcomes fully represent a discrete approximation of the joint distribution of the two uncertainties. The marginal distribution of the first uncertainty determines its percentile realizations. The marginal distributions of both uncertainties, the copula function, and the correlation measure determine the conditional percentile realizations of the second uncertainty. In our framework, a single state of nature tree is constructed for uncertainties within and across different business divisions and time periods, depicting all dependent risks of the firm.

Note that dependencies between risks across different business divisions and across time periods are modeled similarly. Two dependent risks (say, $A$ and $B$ ) from the same time period (say, period 1) will be represented in essentially the same structure as the same risk (say, risk $A$ ) that appears in two time periods (say, periods 1 and 2) and entails dependence from period to period. In the first scenario, risk $A$ is first modeled by the trinomial discretization giving rise to three states of nature. Risk $B$ is then trinomially modeled using conditional uncertainties given each of the three realizations of $A$; hence there are $3^{2}=9$ states for joint realizations of $A$ and $B$. In the second scenario, risk $A$ in period 1 has three possible states of nature, and conditional on this period 1 value, the period 2 risk $A$ value is a trinomially discretized conditional distribution yielding $3^{2}=9$ states of joint realizations of risk $A$ across the two time periods. This copulas-based dependent decision tree framework can be naturally extended in a multivariate time series context to construct a probability tree for sequential, correlated uncertainties. Suppose we have a problem with two dependent uncertainties (say, A and B) in both time periods $(t=1,2)$ that determine a payoff or cash flow. Such a problem can be modeled with a sequence of conditional probability distributions of the four correlated uncertainties in a dependent decision tree. Each conditional scenario is modeled by the trinomial discretization at the endpoints of the predecessor uncertainty, leading to a total of $3^{4}=81$ states that describe the joint distribution of these four uncertainties. For instance, after modeling sequentially Risks $\mathrm{A}$ and $\mathrm{B}$ in the first period as previously described, we model Risk A in period 2 conditional on these two preceding uncertainties and then Risk B in period 2 
conditional on all of the three preceding uncertainties. Multiple risks that appear in multiple time periods are modeled in a similar fashion, as described previously. This is reasonable given that the decision tree approach is a full contingent planning process with natural backward dynamics for modeling dependent uncertainties. Of course, the potential choices of copula functions and correlation measures can be different in different scenarios but the tree size grows in the same way. We referred the interested readers to Wang and Dyer (2012) for more detailed discussions on a multivariate sequential probability tree and methods to consolidate multiple uncertainties into one single uncertainty in each period in order to reduce the size of the constructed decision tree in the proper situation.

An example of such a corporate state of nature tree is presented in the context of our illustrative example in Section 3. A technical description of the dependent decision tree approach is presented in the Appendix for the normal-copula and the $t$-copula. Wang and Dyer (2012) and Clemen and Reilly (1999) provide detailed discussions of the different approaches.

\subsection{Cash Flow Characterization of the Corporate Project Portfolio}

Building on the risk environment described by the dependent state of nature tree, we model the corporate decisions by characterizing cash flows in each state of nature contingent on each possible risk realization and the previous decisions, while considering various constraints. Given the investment opportunities and risk exposures, each division $d$ 's decision making process is modeled by inserting its decisions $X_{d, s}$, into division $d$ 's share of the corporate state of nature tree. We then consolidate the multiple division-level decisions to obtain cash flows from the corporate project portfolio and derive corporate level optimal strategies. Figure 1 (right side) illustrates this process.

Note that cash flows depend on the pertinent risk factors, the required investments, and the dynamic decisions, and thus are uncertain at the end of the planning horizon. Cash outflows usually include the investment, various other associated costs (such as the cost of risk management as described in the next section), and necessary interest and principal payments if borrowing is allowed. Cash inflows primarily depend on the payoffs in the given state of nature. Based on the specific scenario, the payoffs can take on a variety of functional forms. For example, payoffs from financial portfolios may follow a (discretized) normal distribution whereas payoffs from real project investments can be described by a fat-tailed distribution according to the nature of the project. Losses might be captured by skewed and/or fat-tailed distributions. We exhibit incorporating assessed payoff functions into the model in Section 3.

Implementing such a model is challenging due to the quickly accumulating dimensionality associated with the multi-division, multi-dependent-risk, and multi-period environment. To reduce dimensionality, we adapt and further develop the approach in Gustafsson and Salo (2005). Instead of branching out an additional set of decision nodes from the original state nodes as is usually done in decision tree modeling, we leverage a set of logical consistency and capital resource constraints to map these decision variables directly onto the corporate state of nature tree without adding any

new node (and hence not increasing dimensions). Therefore, the size of the decision tree stays the same as the corporate state of nature tree rather than growing in an exponential manner. These 
constraints are discussed next.

\subsection{Model Constraints}

Building upon the cash flow characterization in the corporate tree structure, we can write out the corporate optimization problem, with the objective function and three types of constraints: (1) logical consistency constraints, (2) capital resource constraints, and (3) risk constraints. An example on how to formulate these constraints is given for the hypothetical company in Section 3.

\subsubsection{Logical consistency constraints}

Following the real options modeling approach, we use binary decision variables for investment decisions. The investment decisions made within the division have to be consistent throughout the entire capital budgeting horizon and logical constraints are used to ensure this. For instance, a project can either be selected or not be selected; and if the project is not started in the initial period, the project cannot be continued at a later period. If a decision is required in a given state $s$, the decision maker can take one of the two mutually exclusive and collectively exhaustive binary actions $\left(X_{d, s, Y}, X_{d, s, N}\right)$ for "Yes" (affirmative) and "No" (negative) decisions, i.e., $X_{d, s, Y}+X_{d, s, N}=1$, when the decision in the preceding state pre $(s)$ is affirmative (i.e., $\left.X_{d, p r e(s), Y}=1\right)$. Otherwise, the decision maker does not get to make a decision in state $s$ so neither action can be selected. These logical requirements imply the following consistency constraints across time periods: $X_{d, s, Y}+X_{d, s, N}=$ $X_{d, p r e(s), Y}$. Therefore, each project initiation investment decision is described by a pair of decision variables $\left(X_{d, s, Y}, X_{d, s, N}\right)$ and a corresponding consistency constraint. Each project continuation investment decision entails an additional constraint to maintain consistency with the initial decision. Multiple pairs of such binary decision variables are used if multiple projects are considered.

The use of binary actions and consistency constraints allows us to accomplish dimensionality reduction. Since only an affirmative investment decision will entail cash flows and lead to an uncertain capital position, all unnecessary decision paths associated with a negative decision can be eliminated to conserve dimensionality. Consequently, the size of the decision tree is the same as the size of the state of the nature tree $\left(3^{m}\right.$ for $m$ dependent uncertainties, per the trinomial discretization of each uncertainty), without the multiplier of $2^{k}$ for $k$ pairs of binary decision variables 7 The consistency constraints are also useful in facilitating cash flow characterizations in the capital resource constraints discussed below.

\subsubsection{Capital resource constraints}

Capital resource constraints are employed to ensure that capital is sufficient for future investments and costs of risk management. The capital resource position $V_{s}$ for the corporate project

\footnotetext{
${ }^{7}$ Dimensionality is still an issue for any large scale optimization problem, even with our dimension reduction techniques. As more risks, business divisions, and time periods are considered in the ERM model, dimensionality inevitably increases, as is the case with other methods such as simulations or closed-form option pricing methods. By applying our dimensionality reduction technique, however, we reduce the tree size significantly, thus reducing complexity and computation time. When facing computational challenges in the case of very large numbers of divisions and risks, one intuitive solution is to only consider the most important and heavily dependent risks in the ultimate modeling framework. This is similar to the simplifications needed in any other model-assisted strategic decision-making model of high dimensionality.
} 
portfolio in each state $s$ is obtained by characterizing the cash flows $C F$ in state $s$ (e.g., investment cost, risky returns, and losses), contingent on each division's affirmative investment decisions $X_{d, s, Y}$ and $X_{d, p r e(s), Y}$ :

$$
\begin{gathered}
V_{0}=\sum_{d} C F\left(X_{d, s, Y}, s=0\right)+I_{0} \\
V_{s}=\sum_{d}\left(C F\left(X_{d, s, Y}, X_{d, p r e(s), Y}, s \in S \backslash 0\right)\right.
\end{gathered}
$$

where $C F\left(X_{d, s, Y}, X_{d, p r e(s), Y}, s\right)$ is the cash flow in state $s$ by decisions $X_{d, s, Y}$ and $X_{d, p r e(s), Y}, V_{s}$ is the capital resource position in state $s$, and $I_{0}$ is the initial capital budget. Note that only affirmative investment decisions will impact the cash flows and the corresponding capital resource positions. Therefore, only these decisions need to be modeled in this constraint set further reducing the dimensionality of the problem. Once the cash flows are characterized within the corporate state of nature tree, we can write out the capital resource constraints to capture the cash flow development and end of period capital resource positions.

\subsubsection{Risk constraints}

Since we use LSAD as a measure of the downside risk in calculating the expected utility in the objective function (3), we have additional logical requirements represented in the risk constraints. Let $\Delta V_{s}^{+}\left(\Delta V_{s}^{-}\right)$be nonnegative deviation variables that measure how much the capital resource position $V_{s}$ in state $s$ exceeds (falls short of) the expected final capital resource position $E V_{T}$ over all states at time $T$, the risk constraints are

$$
V_{s}-E V_{T}-\Delta V_{s}^{+}+\Delta V_{s}^{-}=0, s \in S_{T}
$$

and thus the expected LSAD at the end of horizon $T$ in Equation (2) can be rewritten as

$$
L S A D_{T}=\sum_{s \in S_{T}} p(s) \Delta V_{s}^{-}
$$

\subsection{Risk Management Strategies}

Our proposed framework incorporates dependence and allows for "natural hedging" opportunities (e.g., see Gründl et al. 2006) among risks and divisions in corporate decision making. In addition, it considers explicit risk management policies including hedging and other commonly used methods (e.g., (re)insurance and risk control techniques) $]^{8}$ If division $d$ in state $s$ hedges a proportion $\alpha_{d, s}$ of its risk with a cost $C_{d, s}\left(\alpha_{d, s}\right)$, the baseline capital resource constraints needs to be modified to reflect the hedging activity, namely to recognize the hedging cost along with the

\footnotetext{
${ }^{8}$ Henceforth, we use the term "hedging" broadly to indicate an active risk management strategy.
} 
investment and the new project cash flows after hedging, or

$$
\begin{gathered}
V_{0}=\sum_{d} C F\left(X_{d, s, Y}, s=0\right)-X_{d, 0, Y} C_{d, 0}\left(\alpha_{d, 0}\right)+I_{0} \\
V_{s}=\sum_{d} C F\left(X_{d, s, Y}, X_{d, p r e(s), Y}, \alpha_{d, p r e(s)}, s \in S \backslash 0\right)-X_{d, s, Y} C_{d, s}\left(\alpha_{d, s}\right)
\end{gathered}
$$

The future project cash flows in Equation (9) reflects cash outflows for investments, $C F_{O}$, and are adjusted to reflect new cash inflows, $C F_{I}$, now that a hedging strategy takes place in the preceding state(s). The new cash inflows, equals a hedged proportion that is no longer subject to any risks and an un-hedged proportion still subject to the original state-dependent risk outcomes. We measure the a priori risk exposure by the expected value of the future risky outcomes, and thus the hedged cash inflow simply equals the hedge ratio times this expected value (i.e., a proxy for the market price of a hedging contract or the actuarial pricing basis of an insurance or risk control policy). This modeling approach is consistent with the existing literature on hedging policy (c.f. Smith and Stulz 1985; Frestad 2009). Therefore, we have

$$
\begin{aligned}
& C F\left(X_{d, s, Y}, X_{d, p r e(s), Y}, \alpha_{d, p r e(s)}, s\right)=C F_{O}\left(X_{d, s, Y}, s\right)+\left(1-\alpha_{d, p r e(s)}\right) C F_{I}\left(X_{d, p r e(s), Y}, s\right) \\
& +\alpha_{d, p r e(s)} E\left(C F_{I}\left(X_{d, p r e(s), Y}, s\right)\right)
\end{aligned}
$$

Note that we allow each division to choose an appropriate risk management method and an associated hedge ratio $\alpha_{d, s}$ given a possibly different $\operatorname{cost} C_{d, s}$ for each risk. For example, a financial services company with both banking and insurance businesses can use various derivative contracts to manage the market and credit risk and use reinsurance to mitigate the actuarial pricing risk. Note that both linear and nonlinear hedging techniques can be used in this constraint with the same set of hedge ratio decision variables but different function forms of the hedged cash flows, entailing potentially different solution techniques. We discuss the nonlinear hedging techniques more in Section 4.

\subsection{Solution Techniques}

Our optimization problem requires standard solution techniques available from the existing operations research literature. Because some decision variables are restricted to integers (in our case the binary integers) while others are continuous variables, the problem is a mixed integer programming (MIP) problem. A wide variety of problems in optimization can be formulated in this standard form, and many approaches have been proposed for solving the problem (e.g. Savelsbergh 1994, Nemhauser and Wolsey 1999, Li and Sun 2006, and Bonami, et al. 2012). For practical considerations, commercially available software programs can often be efficiently used to find a solution for large scale problems efficiently.

\section{ERM Decision Making for a Hypothetical Financial Services Conglomerate}

We now present an illustrative hypothetical example of a conglomerated financial services com- 
pany and use the framework described in Section 2 to build an optimization problem for this company. Without loss of generality, for ease of presentation and notational convenience we will showcase the formulation and features of the framework using a simplified, representative, and tractable setup. Generalizations and extensions concerning alternative scenarios can be relatively easily derived and some of these possibilities will be further discussed in Section 4 .

In this example, we consider a two-period capital budgeting horizon and two main business divisions, for which the corporate decision maker allocates an initial capital budget $I_{0}$ and make subsequent investment and risk management decisions to maximize the mean-risk form of certainty equivalent from the final capital resource positions at the end of the horizon. The banking division generates revenue from issuing loans to individuals and corporations and is exposed to credit risk for uncertain loan loss in period 2 , denoted by $R_{B}$; the insurance division generates revenues from writing personal and commercial lines of insurance and is exposed to underwriting risk for uncertain insurance loss in period 2 , denoted by $R_{C}$. Both divisions also share the market-riskdriven corporate revenue risk in period 1, i.e., variability in loan and insurance premium income for the banking and insurance division, respectively, denoted by $R_{A}$, since market uncertainties are systematically built into the revenue generation processes.9 Table 1 presents the required investments for division d's project $(d=1,2)$ at $t=0$ and $t=1$ and other assumptions for this example.

The three risk exposures are inter-related across business divisions and time periods. Revenue risk is negatively correlated with credit risk as loan defaults tend to increase when economic conditions deteriorate. Market downturns also dampen demand for some lines of insurance and can have a negative impact on insurers in different ways. For example, life insurers are affected due to asset return decreases and existing guarantees; non-life insurers see higher levels of insurance fraud (cf., Insurance Information Institute 2011); liability insurance and insurance related to credit risk are sensitive to a financial crisis/recession. In addition, credit risk and insurance underwriting/pricing risk are positively correlated via shared customers. Actuarial science and insurance literature have provided evidences and offered psychological and biological based explanations for strong predictive power of an individual's credit score in insurance losses, pointing to positive dependence between credit risk and underwriting risk from a shared clientele base (cf., Brockett and Golden 2007, and Ai et al. 2015).

Following the finance and insurance literature (e.g., Rosenberg and Schuermann 2006), we make assumptions on the marginal distributions and the correlation structure of the three risks. We first describe the risks using the scaled Beta distribution that provides modeling flexibility of distributions with a broad set of shapes. The illustrative parameters are specified in Table 2 and the risks are graphed in Figure 2, from which we can see that revenue risk is a bell shaped distribution whereas credit risk and underwriting risk follow right-skewed loss distributions, capturing large but

\footnotetext{
${ }^{9}$ Note a similar breakdown of risk-based capital risk measures used in insurance regulation, e.g., the Risk Based Capital (RBC) formula for life insurance has separated material risks into four major components: asset risk (C1), insurance risk (C2), interest rate risk (C3), and business risk (C4)(Wong 2002). A company is judged on each of the four components.
} 
rare loan losses and catastrophic insured events. This is consistent with typical assumptions in the literature.

The correlation structure among the three risks as previously described is shown in Table 3.10 We follow Rosenberg and Schuermann (2006) to use -0.5 as a benchmark correlation between market-driven revenues risk and credit risk. Although market conditions tend to have an adverse impact on the insurance business as discussed above, underwriting risk can be largely driven by catastrophes independent of market conditions. Therefore, we assume a relative low correlation of -0.2 between revenue risk and underwriting/pricing risk. Lastly, we assume a moderate percentage of shared clientele base between the loan and insurance divisions within the same financial services company, leading to a moderate and positive correlation of 0.5 between credit risk and underwriting risk.

\subsection{Corporate State of Nature Tree}

Given these three dependent risks in the two time periods, we first follow the process described in Section 2 to formulate a dependent corporate state of nature tree. We choose multivariate Normal copulas because of its flexibility and analytical tractability, and discuss the $t$-copula for tail dependence in the next section. Other copula functions can be adopted in a similar fashion.

Figure 3 (the normal copula-based tree on the left) shows the corporate states of nature tree based on the above assumptions, with contingent outcomes noted for each risk in each possible state. For example, the revenues risk in period $1, R_{A}$, is first modeled as a three-branch tree using the trinomial discrete approximation for the distribution of $R_{A}$, with outcomes (in \$millions) 88.87, 94.86 and 101.62 evaluated at the $5^{\text {th }}, 50^{\text {th }}$, and $95^{\text {th }}$ percentiles, and associated probabilities $0.185,0.63$, and 0.185 assigned to these percentiles, respectively. Because the risks and thus the variables describing them are dependent, the credit risk in period $2, R_{B}$, is modeled contingent on the outcomes of $R_{A}$. For example, when the outcome (in \$millions) of revenue risk $R_{A}$ is 94.86 (i.e., the "middle" state of $R_{A}$ realization), the conditional credit risk in period $2, R_{B} \mid R_{A}=94.86$, is modeled as a three-branch tree with outcomes (in \$millions) 55.82, 68.83, and 86.80. The conditional values $55.82,68.83$, and 86.80 are derived by using the corresponding percentiles (of the distribution of $R_{B}$ ) calculated from the correlation information under the copulas decision tree approach. The probabilities assigned to the three discrete states are again $0.185,0.63$, and 0.185 , respectively, under the EP-T method. We can verify from Figure 3 that the relationship between outcomes of $R_{A}$ and $R_{B}$ reflect negative dependence, i.e., a higher outcome of $R_{A}$ is associated with a lower outcome of $R_{B}$, and vice versa. Similarly, the risk $R_{C}$ is modeled contingent on $R_{A}$ and $R_{B}$. The detailed process for deriving these values and constructing the corporate state of nature tree is discussed in the Appendix.

\subsection{Cash Flows from the Corporate Project Portfolio}

Following the classic real options set-up we assume the company has an initial capital budget

\footnotetext{
${ }^{10}$ In the illustrative example, we have made assumptions directly on the product moment correlation structure of the underlying copula. Wang and Dyer (2012), however, discuss how to derive the correlation structure of the underlying copula from available historical data and the use of nonlinear rank order correlation measures as inputs.
} 
of $I_{0}=\$ 30$ millions for potential projects at the beginning of the budgeting horizon, $t=0$, and decides whether to make fixed initial investments, $I n v_{1,0}$ and $I n v_{2,0}$, respectively for divisions 1 and 2, such as market research, initial setup and recruiting costs, initial marketing expenses, etc., in one or both divisions' projects. Intuitively, these investments are of the nature of sunk cost. We assume that these marketing measures need one period to work out and the size of initial investment does not directly impact the revenue. At $t=1$, market conditions are realized, and the company decides whether to continue investing in the project(s), $I n v_{1,1}$ and $I n v_{2,1}$ respectively for divisions 1 and 2, and generating revenues, i.e., selling insurance policies/issuing loans, servicing the policies and the loans, additional personnel and overhead costs, etc. If the company decides to continue the project(s), revenues are generated at $t=2$ from loan and/or insurance policy issuance such as loan revenue and gross premium written, and losses are incurred from loan defaults and adverse insurance underwriting experiences ${ }^{11}$ In this example, we specify the project payoff structure for each division at the end of the budgeting horizon as "revenues minus losses." For numerical presentation simplicity, we assume a common linear functional form for revenues from both divisions $f_{1}(A)=f_{2}(A)=A$ where functions $f_{1}$ and $f_{2}$ describe how the revenue risk impacts the two divisions' revenues respectively and $A$ is the uncertain outcome of revenue risk $R_{A}$, i.e. the banking division and the insurance division generate the same revenues given the same market conditions. Therefore, the payoff for the banking division is $f(A, B)=A-B$ and the payoff from the insurance division is $f(A, C)=A-C$ at $t=2$, where $B$ and $C$ are the uncertain outcomes (losses) of risks $R_{B}$ and $R_{C}{ }^{12}$ The respective capital surplus in each period is assumed to be invested risk-free during both periods.

Given each division's investment opportunities and how risk factors affect payoffs, each division's decision-making process can be represented in a decision tree. Figure 4 shows a representative segment of the decision trees for divisions 1 and 2. If we were to combine in the usual manner the two division-level decision trees (with binary investment decision variables and trinomial discrete approximation for the dependent risks) into a single corporate-level decision tree, the tree size amounts to $2^{4} \times 3^{3}=432$ nodes because there are two divisions, three risks, and two time periods. However, we do not use this brute force approach. To solve our problem we follow the technique of Gustafsson and Salo (2005) to reduce dimensionality of the corporate decision tree to $3^{3}=27$ nodes, where affirmative decisions are directly mapped onto the corporate state of nature tree with the dimension of 27 final state nodes (as in Figure 3). This is only $6.25 \%$ of the original size, i.e., the size of the decision tree stays the same as the state of nature tree rather than growing exponentially with the added decision variables, thereby greatly improving computational efficiency and managerial relevance. Gustafsson and Salo (2005) reports that decision tree models using this

\footnotetext{
${ }^{11}$ Note that for illustration purpose, we model the revenue risk with simplified assumption that the firm will generate revenue at the end of the budgeting period. In reality, the firm can generate partial revenue in each period, in which case the revenue generated from the first period should be compounded with the risk free rate and included in the capital resource constraint of this period. The new end-of-period capital position will then be used in the second period corporate resource constraint.

${ }^{12}$ Note that the payoff function specification has been chosen for simplicity in our formulation as more complicated functional forms add no additional insights here.
} 
dimension reduction techniques for realistic portfolio selection problems can be solved in reasonable time. This efficiency is partly achieved by adding proper constraints. In the next section we describe the set of constraints used to characterize cash flows and to derive corporate-level optimal strategies based on this corporate decision tree structure.

\subsection{Corporate Objective Function and Model Constraints Formulation}

\subsubsection{Logical consistency constraints}

Implementing logical constraints ensures consistency in investment decisions and conserves dimensionality. As in Section 2, a binary indicator $X_{1,0, Y}=1$ (or 0 ) indicates if an affirmative decision is made for division 1 to make initial investment in its project (or not) at $t=0$ and another binary indicator $X_{1,0, N}=1$ (or 0 ) to indicate whether a negative decision is made (or not). The constraint $X_{1,0, Y}+X_{1,0, N}=1$ makes sure that one and only one of the two actions is taken. Similarly, a pair of indicator decision variables $X_{2,0, Y}$ and $X_{2,0, N}$ are created to describe whether or not to make an initial investment in division 2 project at $t=0$, and again, $X_{2,0, Y}+X_{2,0, N}=1$. Additional binary decision variables and corresponding consistency constraints are used to describe continuing investments for both divisions in period 2.

Table 4 shows the binary investment decision variables used in the two-period contingent capital budgeting process and the associated consistency constraints. Note we have three pairs of such decisions for each division in period 2 corresponding to the trinomial discrete approximation of the commonly shared risk $R_{A}$ in period 1 (e.g., $X_{1,2, Y}$ designates an affirmative action by division 1 in the "middle" state of $R_{A}$ outcome). Obviously, the additional consistency requirement in period 2 is that a continuing investment on a project is possible only if this project is initially invested in at $t=0$, i.e., if $X_{d, 0, Y}=1, d=1,2$.

\subsubsection{Capital resource constraints}

We now discuss the capital resource constraints capturing cash flow development in both divisions and obtain the integrated corporate capital positions. These capital constraints are written out in Table 5. For example, an initial investment $\operatorname{Inv}_{1,0}=\$ 7$ million is needed for division 1 project at time 0 , entailing a cash flow amount of $-7(\$$ million $) \times X_{1,0, Y}$. Similarly, a cash flow of $-7(\$$ million $) \times X_{2,0, Y}$ is attributed to division 2 project at time 0 due to investment Inv $_{2,0}$. Therefore, the total cash flow for the entire corporation at $t=0$ is $-7(\$$ million $) \times X_{1,0, Y}-7(\$$ million $) \times$ $X_{2,0, Y}$. Given the initial capital $I_{0}$ and denoting the capital position in the initial state $S_{0}$ at $t=0$ as $V_{0}$, we can write out the capital resource constraint in $S_{0}$ as $V_{0}=I_{0}-\operatorname{Inv}_{1,0} \times X_{1,0, Y}-\operatorname{In} v_{2,0} \times X_{2,0, Y}$. This capital resource constraint thus effectively integrates the two division-level decision trees into a single corporate decision tree at $S_{0}$. Our model formulation relies only on internal financing not external corporate financing, therefore, an additional $V \geq 0$ constraint is required. This constraint can be relaxed by allowing the corporation to borrow funds at a cost. In this case, the capital resource constraints are adjusted by adding borrowing costs as well as interest and principal repayment.

We proceed in the same manner to map out the rest of the corporate decision tree. In each state 
$s$, a capital constraint measures the corporation's capital resource position $V$ taking into account the beginning capital availability and current period cashflows entailed by divisional decisions $X$ 's and risks $R$ 's. As previously described, continuing investments $\operatorname{Inv}_{d, 1}(d=1,2)$ are required at $t$ $=1$ should the firm decide to proceed and uncertain payoffs from division 1 project $A-B$ and division 2 project $A-C$ are generated at $t=2$, where $A, B, C$ are the uncertain outcomes of risks $R_{A}, R_{B}, R_{C}$. Capital surplus $V_{j}$ at the end of the first period $(t=1, j=1,2,3)$ are deposited at the risk free rate. Consequently, three capital constraints are characterized corresponding to the states at $t=1$ entailed by the common risk $R_{A}$ and twenty-seven capital constraints are used corresponding to the states at $t=2$.

Note that the investment payoffs are uncertain, as they are contingent on how the risks play out during the course of the two periods. By capturing the dependence among these risks, we allow the company to address risk considerations by exploiting the benefits of any "natural hedge" opportunities while staying alert to any "catastrophic" risk exposure potentially created by high risk correlation. For example, the model captures significantly higher risk exposure from both divisions in a bad economy due to the interactions between market, credit, and actuarial pricing risks. Understanding such inter-related risk exposures will help the company make better investment decisions and plan for overall risk management, the goal of ERM.

\subsubsection{Risk constraints and objective function}

For our example, we maximize the mean-risk form of the corporate decision maker's preference in Equation (3) with an assumed risk aversion coefficient of 0.5, i.e.,

$$
\max C E=\left[E V_{T}-\lambda L S A D_{T}\right]=E\left(V_{k}\right)-0.5 E\left(\Delta V_{k}^{-}\right)=\frac{1}{27} \sum_{k=1}^{27} V_{k}-0.5 \frac{1}{27} \sum_{k=1}^{27} \Delta V_{k}^{-}
$$

where $s \in\{(i, j)\}, i=1, \ldots, 9$ and $j=1, \ldots, 3$ for the 27 states at $t=2$. Recall that $\Delta V_{s}^{-}=$ $\max \left\{0, E\left(V_{s}\right)-V_{s}\right\}$ describes how much the amount of capital in state $s \in\{(i, j)\}$ falls short of the expected final resource position and $\Delta V_{s}^{+}=\max \left\{0, V_{s}-E\left(V_{s}\right)\right\}$ describes the surplus. Obviously $\Delta V_{s}^{+} \geq 0$ and $\Delta V_{s}^{-} \geq 0$ and only one of the pair in each state $s \in\{(i, j)\}$ can be strictly positive. Thus, a set of risk constraints are entailed in the form of Equation 6 .

\subsubsection{Risk Management Strategies}

Consistent with the literature (cf., Harrington, Mann, and Niehaus 1995), we now incorporate specific risk management strategies in the integrated capital budgeting framework under dependent risks to allow active risk management. For example, a firm can use a forward contract on the S\&P 500 as a proxy for hedging market risks underlying the revenue risk or commodity derivatives impacting revenue streams. In addition, the firm may consider hedging revenue risks with indexlinked hybrid financial instruments such as by taking a short position on industry/sector indexed mutual funds (see Harrington and Niehaus 1999 for an example of the insurance industry) and can also use better market research or proper product design to mitigate revenue risks. A firm can use the spread between LIBOR and OIS to hedge the credit risk for the banking industry (or similarly 
the spread between a rated corporate bond in the industry of interest and the treasury securities of the matching duration), and pro rata reinsurance policy for hedging actuarial pricing/underwriting risk (cf., Cummins and Weiss 2009).

We assume that risk management $\operatorname{cost} C(\alpha)$ takes a linear form $C(\alpha)=c \times$ exposure $\times \alpha$, where $c$ is the unit risk management cost for a risk exposure and $\alpha$ is the hedge ratio. In this example, we design the unit risk management cost $c$ such that it varies depending on the characteristics of the risks. Consistent with the literature (c.f. Smith and Stulz 1985; Harrington, Mann, and Niehaus 1995), the cost is designed to be higher for more skewed risks to reflect that in practice it is more costly to manage risks that are less "Normal" and more "skewed," due to the lack of risk pooling effect or a higher market beta, i.e., more "skewed" risk $R_{B}$ have a higher cost $c$ than risk $R_{C}$. Table 6 presents unit costs for each of the three risks in our example 13 Note that the change of costs of risk management could impact the optimal risk management decisions both in our analysis and in practice. For instance, a very costly hedging strategy for a new insurance product may over-weigh its benefit and leads to a no decision for pursuing such a project. Therefore, a firm should carefully consider these cost assumptions when using our framework to obtain optimal decisions.

The capital resource constraints need to be revised to reflect the costs and benefits of risk management. For example, for revenue risk $R_{A}$, the hedging cost $C_{A}(\alpha)=$ unit cost $c_{A} \times \operatorname{exposure}$ $E(A) \times$ hedge ratio $\alpha_{d, 0}$ is taken out of period 1 capital available for division $d$ if risk management is used by $d$, and payoffs from the project have to be adjusted for the effect of risk management at the end of the budgeting horizon. The new cash flows $A_{d, j}^{\prime}(d=1,2, j=1,2,3), B_{k}^{\prime}$ and $C_{k}^{\prime}(k=1, \ldots, 27)$ now consist of the hedged portion $\alpha_{d, t}$ of the payoff (based on the expected value $E(A), E(B), E(C)$ of the original volatile cash flows), and the un-hedged portion $\left(1-\alpha_{d, t}\right)$ of the payoff still subject to risks as before. Table 7 presents the adjusted capital resource constraints, corresponding to the original ones in Table 5, with adjustment for risk management costs (cash outflow) and payoffs (cash inflows). While not shown here to conserve space, sensitivity analysis on the risk management cost assumptions show that all optimal binary investment decisions stay the same in every tested scenario (while hedging ratios change), suggesting that our results are robust to moderate changes in the hedging cost assumptions. For ease of illustration, we only consider linear hedging (e.g. pro rata reinsurance policy), since incorporation of nonlinear risk management strategy (e.g., Gay et al. 2003) will add to the computational difficulty for our example without necessarily improving the merits of our modeling approach in a fundamental way. We discuss incorporating nonlinear hedging further in Section 4.4 .

\footnotetext{
${ }^{13}$ The assumed hedging costs structure is justified based on the previous literature (c.f. Smith and Stulz 1985; Camino and Cardone 1999; Harrington and Niehaus 1999; Saunders, Cornett, and McGraw 2006; Thornton 2009). Note that we design the risk management cost also to allow for a variety of decision outcomes to showcase our framework in this numerical example. Corporate risk management strategies reduce the probability of lower-tail realizations, the expected costs associated with financial distress and bankruptcy, the agency cost for managers to engage in risk-shifting, and ease a firm's access to credit (Campello et al 2011). Therefore, the associated risk management cost could reflect the management insights of the overall net cost of the hedging strategy and be firm specific (see more discussion on hedging strategies, and associated benefit and cost in Damodaran 2007). In reality, the firm should be able to obtain these costs from the market or its own estimation to input in the optimization and obtain corresponding optimal risk management and investment decisions.
} 


\subsection{Optimal Decisions under the ERM Decision Framework}

\subsubsection{Optimal decisions}

The proposed integrated optimization framework maximizes the decision maker's expected utility over the final capital positions, contingent on the dependent risk and investment scenarios and subject to the logical consistency constraints, capital resources constraints, and risk constraints. Optimal holistic decisions for investment and risk management are obtained for each division in each period and presented in Table 8 .

The optimal decisions in Table 8 show that the company will invest in both the banking and the insurance businesses in both periods. Both divisions do not hedge revenue risk exposure in period 1. In period 2 both divisions choose to incur the cost of risk management and significantly hedge in order to manage risk exposures in the adverse states. The optimal value of the risk-adjusted certainty equivalent calculated from equation 11 is $\$ 51.38$ million, much greater than investing in risk-free assets: $\$ 30 \times(1+6 \%)^{2}=\$ 33.71$ million, yielding an estimated value or excess return of $\$ 51.38-\$ 33.71=\$ 17.67$ million by optimizing the investment and risk management strategies simultaneously.

We next illustrate the importance on capital budgeting decisions of appropriate dependence modeling and simultaneous consideration of risk management, i.e., the value of ERM. We examine three alternative cases: (1) ignoring, by mistake, underlying risk dependencies, (2) forgoing an appropriate hedging strategy, and (3) considering neither risk dependence nor a hedging strategy.

\subsubsection{Effect on optimal decisions of ignoring risk dependencies}

We first discuss when dependencies among the three risks of the two divisions are not recognized or modeled. All other specifications remain the same as before. Reconstructing the decision tree and solving the optimization problem with a (false) assumption of mutually independent risks (i.e., a diagonal covariance matrix), we obtain the new optimal capital budgeting and risk management decisions shown in Table 9.

In this scenario both divisions allocate capital for investments in both periods and end up not managing revenue risk exposure. The banking division heavily controls the credit risk exposure in all states, while the insurance division only controls the underwriting risk exposure in the middle state in period 2.

Given these false "optimal" decisions, the actual CE of $\$ 49.43$ million is calculated under the true assumptions of dependence, which is a significant reduction of $\$ 51.38$ - $\$ 49.43=\$ 1.95$ from the true optimum of $\$ 51.38$ million, yielding an estimated excess value of $\$ 49.53-\$ 33.71=\$ 15.72$ million, a reduction of $11.04 \%(=\$ 1.95 / \$ 17.67)$ due to the model misspecification error. This example highlights the importance of acknowledging and properly modeling the inter-relations among risks across different business divisions in the ERM corporate planning process. Failure to account for risk dependencies or similarly other synergies within the complex architecture of the corporation can lead to under- or over-investment problems, under- or over-management of risks, or even catastrophic consequences. 


\subsubsection{Effect on optimal decisions of forgoing a hedging strategy}

Now we consider what happens when a hedging strategy is not incorporated into the model. All other specifications remain the same as before except now risk management is no longer part of the decision process. Solving the optimization problem without any active risk management, we obtain the new "optimal" investment decisions shown in Table 10. We note that the optimal capital budgeting decision changes for division 1 to not investing in the pessimistic state in period $2\left(X_{1,3, Y}\right.$ now equals 0$)$. The false "optimal" value for the certainty equivalent is reduced to $\$ 47.84$ million, resulting in an estimated excess value of $\$ 47.84-\$ 33.71=\$ 14.13$ million for the chosen strategies, a $20.05 \%(=(17.67-14.13) / 17.67)$ reduction compared to the original ERM holistic optimization.

This example highlights that appropriately chosen hedging strategies, even at a cost, can add value to the firm ${ }^{14}$. Consistent with modern theories on the value of risk management, by mitigating the impact of underlying market, credit, or underwriting risk exposures, risk management not only increases expected cash flow potentials but also prevents loss to firm value resulting from irreparable underinvestment problems.

\subsubsection{Effect on optimal decisions of considering neither risk dependencies nor a hedg- ing strategy}

If we ignore ERM entirely (i.e., overlooking the risk dependencies and forgo the use of any active risk management strategies) when making capital budgeting decisions, the false "optimal" value for the certainty equivalent is reduced to $\$ 48.59$ million, resulting in an estimated excess value of $\$ 48.59-\$ 33.71=\$ 14.88$ million for the chosen strategies, a $15.79 \%$ reduction due to the model misspecification error and forgoing the hedging strategy. These decisions are reported in Table 11.

Note that the value effects of ignoring risk dependencies, forgoing appropriate hedging strategies, and the combination thereof hinge on the underlying dependence structure and corresponding values for hedging. In our example, when dependence among uncertainties is recognized and the decision maker does not actively hedge, the optimal risk adjusted value is $\$ 47.84$ million. If the decision maker ignores the dependence and still does not hedge, the optimal risk adjusted value is overly estimated at $\$ 48.59$ million. The difference results from a combination of diversification effects due to risk independence, which would have arisen if the risks were really independent, and the role of the forgone hedging strategies. In the model considering the dependence between the revenue risk and the credit risk for the banking division, when the market conditions are not favorable, the conditional credit risk is also relatively high hence the expected project payoff ("revenues minus losses") is relatively low. Therefore, the banking division will hedge in this case (hedging ratio=1) and continue with the project in the second period. When the decision maker forgo hedging and thus provides no cushion from the risk dependencies, the decision maker will intuitively become

\footnotetext{
${ }^{14}$ Note that depending on the appropriate risk management instruments and their respective costs, hedging can also be of limited value to a firm. Our results and insights are derived based on our specific assumptions and given the real risk management measures and costs, the firm's optimization might or might not lead to hedging of any risk exposures.
} 
more conservative and choose to discontinue. In contrast, in the model under the false assumption of independence and when no hedging is allowed, the outcomes of credit risk becomes unrelated to the outcomes of the revenue risk, and as a result, the revenue seems more attractive than it actually is when the market conditions are not favorable. Therefore, the banking division will choose to continue the project. This continuation decision is based on the false assumption of independent risk exposures and therefore results in an over estimation of the risk-adjusted optimal value.

Table 12 provide a summary of these alternative sub-optimal non-ERM models compared with the original optimized ERM model. It shows the extent to which the optimal risk adjusted value would be reduced if we ignore the risk dependencies, or forgoing appropriate hedging strategies with or without the dependence modeling. Clearly, overlooking the (positive) risk dependencies will underestimate the corporate level aggregate risk and the use of optimal hedging strategies under the correct dependence assumption can significantly increase the risk-adjusted optimal value of the corporation.

\section{Robustness Analysis and Model Extensions}

\subsection{Tail Dependence}

The concept of tail dependence has been investigated in financial applications, especially in the context of market or credit risk (e.g., Hauksson et al. 2001 and Embrechts et al. 2002, 2003). Tail dependence measures the probability that extreme events happen jointly, with upper/lower tail dependence measuring the probability of positive/negative extreme events. It is especially of interest in our model since it captures large loss events (default events) between different assets/projects and potential nonlinear dependence structure.

The tail dependence measure depends only on the choice of the copula and not the marginal distributions. The normal copula has both upper and lower tail dependencies equal to zero (Cherubini et al. 2004). One copula function that presents positive upper and lower tail dependence is $t$-copula, indicating a tendency for joint extreme events. Both the normal copula and the $t$-copula belong to the elliptical copulas family. When the number of degrees of freedom increases, the $t$ copula converges to the normal copula. For a limited number of degrees of freedom, however, the behaviors of the two copulas are quite different. We refer the interested reader to the Appendix and Wang and Dyer (2012) for more detailed discussion of $t$-copulas in our modeling approach.

To examine the impact of tail dependence on our results, we reconstruct our optimization model using a $t$-copula for tail dependence and compare the results with those obtained previously. The right side of Figure 3 presents the corporate states of nature tree with an underlying $t$-copula, using the same information as presented in the previous tables and now assuming a degree of freedom of 5 for the $t$-copula to indicate relatively strong tail dependence. We reproduce the original Normal copula results to facilitate side by side comparison. We can see from Figure 3 that in contrast with the normal copula-based tree, the $t$-copula tree shows a fatter tail dependence on both sides.

\subsubsection{Tail dependence and optimal decisions}

Based on the $t$-copula state of nature tree, we maximize the corporate decision maker's expected 
utility over the final capital positions just as before using the proposed optimization framework. Holistic optimal decisions for investment and risk management are obtained for each division in each period and presented in Table 13 .

As we can see, while the hedging strategies are different from the normal-copula case, the optimal decisions remain the same and the optimal value for the certainty equivalent is $\$ 51.21$ million, giving an estimated excess value of $\$ 51.21-\$ 33.71=\$ 17.50$ million for the chosen strategies, within $1 \%$ of the value in the normal copula case. This result illustrates the value of hedging in light of the tail dependence in the ERM framework. The optimal ERM results are relatively insensitive to the tail dependence since the firm can adjust hedging strategies to accommodate extra tail dependence, protecting the overall value.

\subsubsection{Tail dependence and optimal decisions forgoing a hedging strategy}

Consistent with the previous case, the optimal decisions are the same and the optimal value for the certainty equivalent is close to the value in the normal copula case. The tail dependence risk is naturally hedged with a "no" decision for the "bad" state for division 1 in period 2 (i.e., $X_{1,3, Y}=0$ ). The results are the same as presented in Table 10. In summary, we derive very similar insights under a $t$-copula based state of nature tree that dependence modeling and the use of appropriate hedging strategies can enhance firm value in a holistic ERM decision-making process.

\subsection{Sensitivity Analysis on Correlation Assessment and Hedging Cost}

As dependence modeling is a major component in our integrated ERM decision framework, correlation assessments are key inputs in our analysis. In practice, correlation assessments can be difficult to estimate accurately. Here we conduct a set of one-way sensitivity analysis and examine the robustness of the optimal decisions to the correlation estimates, i.e., different correlation matrices are employed in the copula-based dependent state of nature tree to construct the subsequent decision model and obtain optimal decisions.

Clemen et al. (2000) find that experts can reasonably assess correlations and these assessments can be improved by learning. Clemen and Reilly (1999) and Clemen et al. (2000) discuss different subjective correlation assessment methods estimating the probability of concordance and conditional fractile, and report 0.25 as the average mean absolute deviation for correlation assessments.

Following this literature, we examine the effect of each individual correlation assessment by perturbing them one at a time in the \pm 0.25 range of the original correlation assessment. Our sensitivity analysis results suggested that the optimal binary investment decisions stay the same in every tested scenario and thus are robust to reasonable assessment errors. Note that the investment decisions are robust partially because we allow active risk management strategies to be used as a mechanism to coordinate and fine tune capital budgeting decisions. Table 14 shows the range of the hedging ratios when we perturb each original correlation assessment within the \pm 0.25 range 15 and the corresponding optimal risk-adjusted value. Without risk management, optimal decisions will be more sensitive to these assessment errors, consequently leading to enterprise decisions that

\footnotetext{
${ }^{15}$ We assume that the expert can at least be certain about the direction of correlation and therefore only perturb the correlation between $B$ and $C$ from -0.2 to 0 (instead of -0.2 to 0.05 ) and -0.2 to -0.45 .
} 
fail to maximize firm value.

In addition, hedging provides benefits at a cost and companies often face uncertainty in hedging costs in the market. Therefore, it is important to test whether optimal decisions are sensitive to changes in hedging costs. We allow each of the three unit hedging costs to inflate or deflate by $20 \%$ and present the sensitivity analysis results in Table 14. Optimal investment decisions stay the same in every tested scenario, suggesting that they are robust to moderate changes in hedging costs. Consistent with our intuition, when the hedging cost is higher, the firm hedges less and the corresponding optimal risk-adjusted value decreases and vice versa.

\subsection{Additional Downside Risk Constraints}

It has become standard practice for banks and financial institutions to calculate value at risk and similar quantile-based risk measures for portfolio risk exposure on a regular basis. Ai et al. (2012) discussed the use of VAR constraints as input into the chance-constrained optimization in the context of ERM decision making and how one could then utilize chance constrained programming techniques for the solution.

In the decision tree model, we may not have a value that exactly matches the lower $\alpha$ percentile. In the discrete setting, Hardy (2006) chooses the closest value for the loss that gives at least a $\alpha$ percentile according to the definition of VaR. Alternatively, we can also simulate the discrete uncertainty with many scenarios to calculate the percentiles, or fit the discrete approximation with a continuous distribution with Kolmogorov-Smirnov goodness of fit or Chi-square goodness of fit test and approximate the $\operatorname{VaR}_{\alpha}(X)$ and the corresponding CVaR with the fitted continuous distribution. With the approximation of $\mathrm{VaR}$ or $\mathrm{CVaR}$, we can add an additional downside risk constraints $\operatorname{VaR}_{\alpha}(X) \geq V a R_{\text {benchmark }}$, or $C V a R_{\alpha}(X) \geq C V a R_{\text {benchmark }}$, where $V a R_{\text {benchmark }}$ or $C V a R_{\text {benchmark }}$ is the corresponding downside risk constraint requirement of the financial institution.

\subsection{Considerations for Practical Applications, Model Extensions, and Limitations}

This paper focused on presenting the conceptualization of an integrated ERM and capital budgeting framework in a computationally feasible formulation. Our approach has modeling flexibilities in accommodating different model assumptions and configurations. Under the same conceptual framework, a few interesting extensions are immediate.

First, we used a mean-risk form overall utility function to model the corporate decision maker's risk preferences. In addition to the mean-LSAD model discussed above, other valid forms of utility functions can be used in the objective function to form the optimization problem as discussed in Gustafsson and Salo (2005) ${ }^{16}$ Alternatively as suggested in the ERM literature (e.g., Ai et al. 2012), the decision maker's risk appetite can be articulated and directly built into the decision pro-

\footnotetext{
${ }^{16}$ We reexamined the constrained optimization framework with the mean-variance setup that is widely used in the financial portfolio selection literature. In this setup, variance is the risk measures used in the objective function and in the constraints. The results using the mean-variance approach change from considering only downside risks (as one might expect) since minimizing variance also entails aversion to large upside potential, which is not present in our mean-LSAD approach. To conserve space, the results are not tabulated and are available upon request.
} 
cess, where the corporate decision maker is allowed to have different preferences and prioritization for different risk types, divisions, projects, and planning horizons. For example, risk appetite modeling could reflect the decision maker's preference for anticipated information and agency problems associated with a particular set of investment opportunities. It could also capture the corporation's risk culture as related to the overall corporate culture, ownership and capital structure, managerial compensation mechanisms, or the regulatory environment.

Second, we focused on linear risk management methods, such as forward/future contracts and pro rata reinsurance contract, and assumed that all active risk management activities are planned as the dynamic decision process goes. Alternatively, we could explore and incorporate non-linear risk management strategies (such as options or excess of loss insurance) that might be appropriate for certain types of risks. These non-linear hedging techniques can be accommodated in our model framework at the cost of added complexity in the constraint set. Essentially non-linear risk management leads to a piecewise functional form of the hedged payoff instead of the linear functional form as shown in the illustrative example. We can incorporate it into our framework using the same decision variables of hedge ratio and cash flow characterizations. For example, we can use the same hedge ratio decision variable to capture the extent of hedging using call option contracts for, say, commodity price fluctuations. The cost of hedging will be the option premium and the cash flows need to be adjusted for according to a piece-wise function representing the payoff structure for options, and the underlying commodity price uncertainty can be modeled in the similar way as we model the other risks. The solution techniques may have to vary in light of the nonlinear constraints entailed by the nonlinear risk management strategy (cf., Nemhauser and Wolsey 1999, Li and Sun 2006, and Bonami, et al. 2012). It will also be relatively easy to allow all risk management decisions to be planned at the beginning of the entire capital budgeting horizon (instead of at the beginning of each period) by modifying the corresponding cash flow characterizations and capital constraints.

Third, we use the copula approach to model dependencies among risks. The copula model can accommodate a large class of dependence structure including the usual Pearson product moment linear correlation, rank order correlation and tail dependence within parametric families of copula functions such as normal copulas, $t$-copulas and Archimedean copulas. While we used a Normal copula and $t$-copula to illustrate the model in the illustrative example presented in Section 3 , the proposed framework provides efficient accommodations for other copula models.

Our goal in this paper is to develop a conceptual framework for integrating important corporate functions as an optimization model recognizing across-the-board dependencies among risk exposures, business divisions, and time periods within a complex modern corporation. To formulate the optimization problem, we leveraged the convenient and efficient tool of decision tree to facilitate dependence modeling. In order to clearly illustrate the modeling approach, we resort to some simplifications presented in the paper. Most extensions beyond the current assumptions, such as those discussed above and many other relatively minor ones not expounded upon here (e.g., different 
forms of payoff functions), should be rather straightforward to incorporate into our optimization model. Although they may require more sophisticated solution techniques, these are abundantly available in the current operations research literature. However, our modeling approach comes with its own set of potential limitations. First, dimensionality is a notable issue for any large scale optimization problem. Our decision tree-based approach is more economical than other approaches (such as large-scale simulations) by carefully choosing a small set of input values and taking advantage of available dimension reduction techniques. However, we still have to address the dimensionality issue as it becomes more severe with overly large managerial problems when the number of points in the approximation increases. Second, any discretization, as we use in the EP-T based decision tree approach, might result in loss of valuable information. Therefore, the decision maker should carefully trade off the desire for managerial flexibility and parsimony against accuracy and thoroughness. Third, the enhancement of certain modeling components, such as the incorporation of complex nonlinear hedging techniques and risk appetite modeling, may lead to new challenges in finding a feasible solution in an affordable fashion. Last but not least, as is true with other modeling approaches, the appropriate selection of parameter values is important in implementing our modeling framework in order to derive useful results and insights. We discuss more challenging future research avenues in the conclusion.

\section{Conclusion}

This paper proposed an integrated capital budgeting and ERM framework under dependent risks in a multi-division, multi-project and multi-period environment. We formulated the model as an optimization problem of the corporate decision maker and constructed the model via a decisiontree interface. We illustrated our framework using a hypothetical financial services company.

Rather than maintaining two separate functions for risk management and capital budgeting for investments as traditionally done in the literature, these corporate decisions are aligned under a common set of strategic objectives and risk preferences. As business divisions are intrinsically connected by a set of dependent risk exposures and possibly other synergetic relationships, the integrated framework ensures consistency in divisional decisions and facilitates achieving the enterprise goals. In accomplishing this task, a prototype modeling approach was proposed to assist in conceptual formulation and can also overcome several modeling and computational challenges, such as dependence modeling and the increased dimensionality.

Our paper contributes to the growing literature on enterprise risk management by prescribing such an integrated corporate decision framework that explicitly accounts for the shared and dependent risks from inter-related business divisions, a problem of particular challenge and importance for financial conglomerates. We address a scarcity of research in the ERM literature concerning quantitative (as opposed to qualitative) descriptions of holistic modeling and the possible benefits from such formulations, including hedging effects. Our paper also has significant managerial relevance for corporate decision-making in practice. Decision makers can adapt our model efficiently under their own specifications. 
The proposed integrated framework opens future research avenues for further improvements and new issues. For example, we have followed the literature to consider binary investment decisions with a given capital requirement in the current framework (e.g., Gustafsson and Salo 2005). There might be times when an investment decision is desired to be with more than two alternatives. There may also be times when the decision maker wishes to build sub-module problems before inputting them into the corporate level problem in a multi-stage sequential optimization, especially when the size of the corporate level optimization is overly large for one single model. In addition, the current framework only captures inter-related risk dependencies across business divisions and time periods. There may be other synergies within a corporation that are similar from a modeling perspective, for example, benefits and costs from research and development initiatives, marketing campaigns, and fixed capital expenditures that are shared by multiple business divisions. Moreover, besides the integration of corporate decisions on capital budgeting and risk management, external corporate financing policies could also be incorporated into the current framework so that all the three main areas of corporate functions are aligned at the enterprise level. Finally, the proposed integrated framework can be extended in light of the new strand of behavioral economics literature where the decision maker's behavioral biases are considered in the choice of utility function or risk preference modeling. These interesting future research topics can be built upon the same conceptual and technical grounds of our current modeling framework. 


\section{References}

Ai. J., P.L. Brockett, L.L. Golden, and B. Kellison, 2015, Empirical Evidence on the Use of Credit Scoring for Predicting Insurance Losses with Psycho-Social and Biochemical Explanations, working paper.

Ai, J., P. L. Brockett, W. W. Cooper, and L. L. Golden, 2012, Enterprise Risk Management through Strategic Allocation of Capital, Journal of Risk and Insurance, 79:29-56.

Ang, A., J. Chen, and Y. Xing, 2006, Downside Risk, Review of Financial Studies, 19:1191-1239.

Beasley, M., D. Pagach, and R. Warr, 2008, Information Conveyed In Hiring Announcements of Senior Executives Overseeing Enterprise-wide Risk Management Processes,. Journal of Accounting, Auditing E Finance, 23(3):311-332.

Bernardo, A., H. Cai, and J. Luo, 2004, Capital Budgeting in Multi-Division Firms: Information, Agency, and Incentives, Review of Financial Studies, 17:739-767.

Bonami, P., M. Kilinç, and J. Linderoth, 2012, Algorithms and software for convex mixed integer nonlinear programs. In Mixed Integer Nonlinear Programming. Springer New York.

Brealey, R.A., and S.C. Myers, 2003, Principles of Corporate Finance, 7th ed. McGraw-Hill Irwin, New York.

Brockett, P. L., and L. L. Golden, 2007, Biological and Psychobehavioral Correlates of Credit Scores and Automobile Insurance Losses: toward an Explication of Why Credit Scoring Works, Journal of Risk and Insurance, 74:23-63.

Campello, M., Lin, C., Ma, Y., and Zou, H. 2011. The Real and Financial Implications of Corporate Hedging. Journal of Finance, 66(5), 1615-1647.

Camino, D., and C. Cardone. 1999. The Valuation and Cost of Credit Insurance Schemes for SMEs: the Role of the Loan Guarantee Associations. International Small Business Journal, 17(4), 13-31.

Cherubini, U., E. Luciano, and W. Vecchiato, 2004, Copula Methods in Finance, New York: John Wiley \& Sons Ltd.

Clemen, R. T., and T. Reilly, 1999, Correlations and Copulas for Decision and Risk Analysis, Management Science, 45:208-224.

Clemen, R. T., G. W. Fisher, and R. L. Winkler, 2000, Assessing Dependence: Some Experimental Results, Management Science, 46:1100-1115.

Cummins, J. D. and M. A. Weiss, 2009. Convergence of Insurance and Financial Markets: Hybrid and Securitized Risk-Transfer Solutions. Journal of Risk and Insurance, 76: 493-545.

Committee of Sponsoring Organizations (COSO), 2004, Enterprise Risk Management-Integrated Framework: Executive Summary, COSO, New York.

Damodaran, A. 2007. Strategic Risk Taking: a Framework for Risk Management. Pearson Prentice Hall.

Deaton, A., 1981, Optimal Taxes and the Structure of Preferences, Econometrica: Journal of the Econometric Society, 49(5):1245-1260.

Embrechts, P., A. McNeil, , and D. Straumann, 2002, Correlation and Dependency in Risk Management: Properties and Pitfalls, Risk Management: Value at Risk and Beyond, 176-223.

Embrechts, P., F. Lindskog, and A. McNeil, 2003, Modelling Dependence with Copulas and Applications to Risk Management, Handbook of Heavy Tailed Distributions in Finance, 8(1): 329-384.

Embrechts, P., 2009, Copulas: A Personal View, Journal of Risk and Insurance, 76(3):639-650.

Eppen, G. D., R. K. Martin, and L. Schrage, 1989, A Scenario Approach to Capacity Planning, Operations Research, 37:517-527.

Fishburn, P. C., 1977, Mean-risk Analysis with Risk Associated with Below Target Returns, American Economic Review, 67:116-126.

Frees, E. W., and E. A. Valdez, 1998, Understanding Relationships Using Copulas, North American Actuarial Journal, 2(1):1-25.

Frestad, D., 2009, Why Most Firms Choose Linear Hedging Strategies, Journal of Financial Research, $32(2): 157-167$.

Froot, K.A., D.S. Scharfstein, and J.C. Stein, 1993, Risk Management: Coordinating Corporate Investment and Financing Policies, Journal of Finance, 48:1629-1658.

Froot, K., and J. C. Stein, 1998, Risk Management, Capital Budgeting, and Capital Structure Policy for Financial Institutions: An integrated approach, Journal of Financial Economics, 47:55-82. 
Froot, K. A., 2007, Risk Management, Capital Budgeting, and Capital Structure Policy for Insurers and Reinsurers, Journal of Risk and Insurance, 74(2):273-299.

Gatzert, N., A. Kolb. 2014. Risk Measurement and Management of Operational Risk in Insurance Companies from an Enterprise Perspective. Journal of Risk and Insurance, 81(3), 683-708.

Gatzert, N., M. Martin, 2015. Determinants and Value of Enterprise Risk Management: Empirical Evidence from the Literature. Risk Management and Insurance Review, 18(1), 29-53.

Gay, G. D., J. Nam, and M. Turac, 2003, On the Optimal Mix of Corporate Hedging Instruments: Linear Versus Nonlinear Derivatives. Journal of Future Market, 23(3):217-239.

Grace, M. F., J. T. Leverty, R. D. Phillips, and P. Shimpi, 2015, The Value of Investing in Enterprise Risk Management, Journal of Risk and Insurance, 82(2), 289-316.

Gollier, C. 2001, The Economics of Risk and Time. MIT Press, Cambridge, MA.

Gründl, H., and H. Schmeiser, 2007, Capital Allocation for Insurance Companies-What Good IS IT? Journal of Risk and Insurance, 74(2):301-317.

Gründl, H., T. Post, and R. N. Schulze, 2006, To Hedge or Not to Hedge: Managing Demographic Risk in Life Insurance Companies, Journal of Risk and Insurance 73(1):19-41.

Gustafsson, J., and A. Salo, 2005, Contingent Portfolio Programming for the Management of Risky Projects, Operations Research, 53:946-956.

Hammond, R.K, and J.E. Bickel, 2013, Reexamining Discrete Approximations to Continuous Distributions, Decision Analysis, 10(1): 6-25.

Hammond, R. K., 2014, Discrete Approximations to Continuous Distributions in Decision Analysis, Doctoral dissertation.

Hauksson, H., M. Dacorogna, T. Domenig, U. Mueller, and G. Samorodnitsky, 2001, Multivariate Extremes, Aggregation and Risk Estimation, Quantitative Finance, 1:79-95.

Hardy, M. R. 2006, An Introduction to Risk Measures for Actuarial Applications, SOA Syllabus Study Note.

Harrington, S. E., S. V. Mann, and G. Niehaus. 1995, Insurer Capital Structure Decisions and the Viability of Insurance Derivatives. Journal of Risk and Insurance, 62(3): 483-508.

Harrington, S., and G. Niehaus. 1999. Basis risk with PCS catastrophe insurance derivative contracts. Journal of Risk and Insurance, 49-82.

Hoyt, R. E., and A. P. Liebenberg, 2011, The Value of Enterprise Risk Management, Journal of Risk and Insurance, 78(4):795-822.

Insurance Information Institute, 2011, Insurance Fraud/http://www2.iii.org/insuranceindustry/fraud/ Accessed May 2, 2011.

Keefer, D. L., and S. E. Bodily., 1983, Three-Point Approximations for Continuous Random Variables, Management Science, 29:595-609.

Keefer, D.L., 1994, Certainty Equivalents for Three-Point Discrete-Distribution Approximations, Management Science, 40:760-773.

Laeven, R.J.A., and M. J. Goovaerts, 2004, An Optimization Approach to The Dynamic Allocation of Economic Capital, Insurance: Mathematics and Economics, 35(2): 299-319.

Li, D., and X. Sun, 2006. Mixed-Integer Nonlinear Programming. Nonlinear Integer Programming, Springer.

Lin, Y., M. Wen, and J. Yu, 2012, Enterprise Risk Management: Strategic Antecedents, Risk Integration, and Performance, North American Actuarial Journal, 16(1):1-28.

Longstaff, F. A., and E. S. Schwartz, 2001, Valuing American Options by Simulation: A Simple Least-squares Approach, Rev. Financial Stud, 14(1):113-147.

Massey Jr, F. J., 1951, The Kolmogorov-Smirnov Test for Goodness of Fit, Journal of the American statistical Association, 46(253):68-78.

Myers, S. C., and Jr, J. A. Read, 2001, Capital Allocation for Insurance Companies, Journal of Risk and Insurance, 68)4):545-580.

Natarajan, K., D. Pachamanova, and M. Sim., 2009, Constructing Risk Measures from Uncertainty Sets, Operations Research, 57:1129-1141.

Nemhauser, G.L., and L. A. Wolsey, 1999, Integer and Combinatorial Optimization, New York: Wiley.

Nelsen, R.B., 1999, An Introduction to Copulas, New York: Springer.

Obersteadt, Anne et al. 2013 State of the Life Insurance Industry: Implications of Industry Trends Center for Insurance Policy and Research White Paper, National Association of Insurance Commissioners 
Pagach, D., and R. Warr, 2011, The Characteristics of Firms That Hire Chief Risk Officers, Journal of Risk and Insurance, 78(1):185-211.

Pencavel, J. H., 1979, A Note on Income Tax Evasion, Labor Supply, and Nonlinear Tax Schedules, Journal of Public Economics, 12(1):115-124.

Rosenberg, J., and T. Schuermann, 2006, A General Approach to Integrated Risk Management with Skewed, Fat-tailed Risks, Journal of Financial Economics, 79:569-614.

Saunders, A., M. M. Cornett, and P. A. McGraw. 2006. Financial institutions management: A risk management approach (Vol. 8). McGraw-Hill/Irwin.

Savelsbergh, M.W.P., 1994, Preprocessing and Probing Techniques for Mixed Integer Programming Problems, ORSA J. Computing, 6(4): 445-454.

Sherali, H.D., E. Dalkiran, and T. S. Glickman, 2011, Selecting Optimal Alternatives and Risk Reduction Strategies in Decision Trees, Operations Research 59:631-647.

Smith, C. W., and R. M., Stulz, 1985, The Determinants of Firms' Hedging Policies, Journal of Financial and Quantitative Analysis, 20(4):391-405.

Smith, J. E. 1993. Moment Methods for Decision Analysis. Management Science, 39(3), 340-358.

Smith, J. E., 2005, Alternative Approaches for Solving Real-options Problems: (Comment on Brandão et al. 2005), Decision Anal., 2(2):89-102.

Smith, A. E., P. B. Ryan, and J. S. Evans, 1992, The Effect of Neglecting Correlations When Propagating Uncertainty and Estimating the Population Distribution of Risk, Risk Analysis, 12:467-474.

Smith, J. E., and R. F. Nau, 1995, Valuing risky projects: option pricing theory and decision analysis, Management science, 41(5):795-816.

Thornton, D. L. 2009. What the Libor-OIS spread says. Economic Synopses.

Tsetlin, I, R.L. Winkler, 2005, Risky Choices and Correlated Background Risk, Management Science, 51:1336-1345.

Wang, T., and J. S. Dyer, 2010, Valuing Multifactor Real Options using an Implied Binomial Tree, Decision Anal., 7(2):185-195.

Wang, T., and J. Dyer. 2012, A Copulas-based Approach to Modeling Dependence in Decision Trees, Operations Research, 60:225-242.

Wang, T., J. S. Dyer, and J. C. Butler, 2015, Modeling Correlated Discrete Uncertainties in Event Trees with Copulas, Risk Analysis, 2015, DOI: 10.1111/risa.12451

Zhang, Y., and V. Dukic, 2012, Predicting Multivariate Insurance loss payments under the Bayesian copula framework, Journal of Risk and Insurance, 80(4):891-919. 


\section{Appendix. Normal-copula based Dependent Decision Tree and an Extension to t-copula}

In the illustrative example presented in the paper, we use the Normal copulas-based dependent decision tree for its simplicity and its suitability to construct our optimization framework. A brief introduction to the Normal copulas-based dependent decision tree approach is presented below. A multivariate Normal copula $C_{n}$ is given by $C_{n}\left(u_{1}, \ldots, u_{n}\right)=\Phi_{\Sigma}\left(\Phi^{-1}\left(u_{1}\right), \ldots, \Phi^{-1}\left(u_{n}\right)\right)$. It is derived from a multivariate normal cumulative distribution function $\Phi_{\Sigma_{Z}}$ with mean zero and correlation matrix $\Sigma$, by transforming the uniform random variables $\left(u_{1}, \ldots, u_{n}\right)$ on $[0,1]$ by taking the inverse of the standard Normal distribution function $\Phi$. With the information of marginal and assessed pairwise correlations, there are two steps to construct the normal copula-based dependent decision tree.

Step 1. Construction of the normal copulas based transient tree structure. Since copula is independent of the choice of marginal distribution, we can focus on the probability tree for discrete approximations of underlying normal copula with uniform variables first, and combine it with the information of marginals in the next step. To build the multivariate Normal copula-based decision tree for variables $\left(X_{1}, \ldots, X_{n}\right)$, we first construct a discrete approximation for the unconditional Uniform variable $u_{1}$, and then recursively compute the dependent Uniform variables $u_{k}(k=2, \ldots, n)$,conditioning on each of the point realizations of the previous discrete approximations for $\left(u_{1}, \ldots, u_{k-1}\right)$. Thus, $u_{n}$ can be written as

$$
u_{n=} \Phi\left(A_{n 1} \Phi^{-1}\left(\alpha_{1}\right)+\ldots+A_{n(n-1)} \Phi^{-1}\left(\alpha_{n-1}\right)+A_{n(n)} \Phi^{-1}\left(\alpha_{n}\right)\right),
$$

where $A_{n j}$ is the $(n, j)$ element of the Cholesky factorization that decomposes the covariance matrix $\Sigma$ as $\Sigma=A A^{T}$ to give the lower triangular matrix $A=\left(A_{i j}\right)_{i, j=1}^{n}$ and $\alpha_{i}$ is the predetermined (optimally chosen) percentiles of the conditional distribution $u_{i} \mid u_{1}, \ldots, u_{i-1}$ according to the extended Pearson-Tukey method (Keefer and Bodily, 1983).

Step 2. Point-to-point inverse marginal transformation. After the calculation of $u_{i}$, we are ready to combine the marginal information and transform them to obtain the discrete approximations to the original uncertainties. The discrete approximations of $X_{i}$ are obtained by applying the inverse of the target marginal distribution function for each realization of $u_{i}$, i.e., $X_{i}=F_{i}^{-1}\left(u_{i}\right)$. Taking the point-to-point inverse marginal transformation, each realization of $\left(X_{1}, \ldots X_{n}\right)$ is discretely approximated using the extended Pearson-Tukey method. Similarly, we create the contingent tree for each successive node until we generate the complete multivariate standard decision tree for $\left(X_{1}, \ldots, X_{n}\right)$. Throughout the tree, the point realizations of these approximations vary with the conditioning scenarios; the probabilities assigned for the three branches of a chance node do not vary with the conditioning scenarios using the logic of the EP-T approximations.

Note that because the multivariate normal distribution $\Phi_{\Sigma_{Z}}$ for the normal copula is parameterized in terms of a product moment correlation $\Sigma_{Z}=\left(r_{i j}\right)_{i, j=1}^{n}$, we need to calculate the corresponding Pearson product moment correlation $r_{i j}$ for each pair of correlations of the original random variables $X_{1}, \ldots, X_{n}$. For illustration purpose, we have made assumptions on the correlation structure of the underlying copula directly and refer the interested readers to Wang and Dyer 
(2012) on how to derive the correlation structure of the underlying copula from available historical data.

We now illustrate this approach in the context of our example presented in Section 3. Our goal is to model the dependence structure of the three risks $R_{A}, R_{B}$, and $R_{C}$ in the decision tree. To create the probability tree for the standardized Uniform variables, we first generate the extended Pearson-Tukey discretization for $u_{A}$. It is a three point discrete approximation for the standard Normal distribution with probabilities $0.185,0.630$, and 0.185 assigned to the percentiles $0.05,0.5$ and 0.95. The three discretized possible outcomes of the risk $R_{A}$ are therefore (\$million) 88.87, 94.86 and 101.62, the inverse at the $5^{\text {th }}, 50^{\text {th }}$, and $95^{\text {th }}$ percentile of the Beta distribution describing $R_{A}$, respectively.

Due to the dependence among the three risks $R_{A}, R_{B}$, and $R_{C}$, the subsequent discrete approximations for $R_{B}$, and $R_{C}$ are contingent on the outcomes of $R_{A}$. We first apply the Cholesky factorization to decompose the correlation matrix $\Sigma_{Z}$ given in Table 3 into the lower triangular Cholesky matrix shown in Table 15 to assist the calculation of the dependent uniform variables.

(1) The dependent uniform $u_{B}$ given the outcomes of $u_{A}$. Using formula (A1) for the bivariate case, we can calculate $u_{B}$ as follows:

$$
u_{B}=\Phi\left(-0.5 \Phi^{-1}\left(\alpha_{1}\right)+0.866 \Phi^{-1}\left(\alpha_{2}\right)\right) \text {, }
$$

(2) The dependent uniform $u_{C}$ given the outcomes of $u_{A}$ and $u_{B}$. Using formula (A1), $u_{C}$ is calculated as follows:

$$
u_{C}=\Phi\left(-0.2 \Phi^{-1}\left(\alpha_{1}\right)+0.462 \Phi^{-1}\left(\alpha_{2}\right)+0.864 \Phi^{-1}\left(\alpha_{3}\right)\right)
$$

For instance, when the outcome of $u_{A}$ is 0.5 (i.e., the $50^{\text {th }}$ percentile), the conditional distribution for $u_{B} \mid u_{A}=0.5$ is calculated for the $5^{t h}, 50^{t h}$, and $95^{t h}$ percentiles using (A2), yielding the three contingent outcomes of $u_{B}$ to be $0.077,0.5$, and 0.923 , respectively. Therefore, the three discrete outcomes of the risk $R_{B}$ given $R_{A}=94.86$ (\$million) are therefore (\$million) 55.82, 68.83 and 86.80, the inverse of the conditional distribution for $R_{B}$ at the $5^{t h}, 50^{t h}$, and $95^{t h}$, respectively. Similarly, using (A3) we generate the three point discrete approximation for risk $R_{C}$ dependent on $R_{A}$ and $R_{B}$.

At the end of period 1, there are three possible states for revenue risk $R_{A}$ outcomes corresponding to the trinomial (unconditional) discrete approximation of $R_{A}$. At the end of period 2, i.e., the end of the capital budgeting horizon, there are a total of 27 states, corresponding to the trinomial (unconditional) discrete approximation of $R_{A}$, the nine $\left(3^{2}=9\right)$ possible states taking into account the dependent pair of risks $\left(R_{A}, R_{B}\right)$, and the twenty seven $\left(3^{3}=27\right)$ possible final states taking into account the dependent triplet of risks $\left(R_{A}, R_{B}, R_{C}\right)$.

If we have more than three risks, we follow a similar fashion to derive the dependent uniform variables based on (A1), calculate the conditional discrete approximations of the risks, and build the tree structure with the desired marginal distributions and dependencies of the risks.

Our proposed framework also provides efficient accommodations for $t$-copulas and Archimedean copulas to capture tail dependence when extreme events occur. A multivariate $t$-copula $C_{n}$ is given by $C_{T}\left(u_{1}, \ldots, u_{n}\right)=t_{\Sigma, \nu}\left(t_{\nu}^{-1}\left(u_{1}\right), \ldots, t_{\nu}^{-1}\left(u_{n}\right)\right)$, where $t_{\nu}$ is the univariate student's t distribution 
function, with $\nu$ degrees of freedom, and $t_{\Sigma, \nu}$ the multivariate distribution corresponding to $t_{\nu}$. To build the multivariate $t$-copula-based decision tree for variables $\left(X_{1}, \ldots, X_{n}\right)$, we first construct a discrete approximation for the unconditional uniform variable $u_{1}$, and then recursively compute the dependent uniform variables $u_{k}(k=2, \ldots, n)$, conditioning on each of the point realizations of the previous discrete approximations for $\left(u_{1}, \ldots, u_{k-1}\right)$. Thus, $u_{n}$ can be written as

$$
u_{n=} t_{\nu}\left(A_{n 1} t_{\nu}^{-1}\left(\alpha_{1}\right)+\ldots+A_{n(n-1)} t_{\nu}^{-1}\left(\alpha_{n-1}\right)+A_{n(n)} \sqrt{\frac{\nu+\left[t_{\nu}^{-1}\left(\alpha_{1}\right)\right]^{2}}{\nu+1}} t_{\nu+1}^{-1}\left(\alpha_{n}\right)\right),
$$

where $A_{i j}$ is the element of the Cholesky factorization that decomposes the covariance matrix $\Sigma$ as $\Sigma=A A^{T}$ to give the lower triangular matrix $A=\left(A_{i j}\right)_{i, j=1}^{n}$ and $\alpha_{i}$ is the percentile of the conditional distribution $X_{i} \mid X_{1}, \ldots, X_{i-1}$. We follow essentially the same process for the normalcopula case to develop the $t$-copula based dependent decision tree now using equation (A4) instead of (A1). We refer the interested readers to Wang and Dyer (2012) for detailed discussions of the dependent decision tree approach. 